\title{
Effects of three-area laser-assisted zona thinning in 8-cell human embryos on pregnancy outcomes in vitro fertilization
}

\author{
Ju-Eun Jeong ${ }^{1}$, Bo-Sun Joo ${ }^{2}$, Chang-Woon Kim ${ }^{3}$, Hwi-Gon Kim¹, Jong-Kil Joo', Kyu-Sup Lee ${ }^{1}$ \\ 'Department of Obstetrics and Gynecology, Medical Research Institute, Pusan National University School of Medicine, Busan; ${ }^{2}$ Healthy Aging Korean \\ Medical Research Center, Pusan National University, Busan; ${ }^{3}$ Department of Obstetrics and Gynecology, Samsung Changwon Hospital, Sungkyunkwan \\ University School of Medicine, Changwon, Korea
}

Objective: This study conducted a preliminary examination of the effects of three-area laser-assisted zona thinning (LAZT) during the cleavage stage of embryo development on the hatching process in human in vitro fertilization-embryo transfer (IVF-ET) with subjects of advanced female age or frozen-thawed (FT) embryos.

Methods: Eight-cell stage embryos were treated with LAZT in three areas of the zona pellucida at $120^{\circ}$ intervals. The control group was embryos without LAZT. Of the 72 consecutive fresh cycles and the $28 \mathrm{FT}$ embryo transfer cycles, the patients in 55 fresh cycles and $17 \mathrm{FT}$ cycles declined LAZT, and those cycles were defined as the control group.

Results: In the fresh cycles, the pregnancy rates were similar in the LAZT and control groups. However, in the FT cycles, the pregnancy rate was significantly higher in the LAZT group than in the control group ( $45.5 \%$ in the LAZT group vs. $23.5 \%$ in the control group, $p<0.05$ ).

Conclusion: These results show that multi-area LAZT resulted in significantly improved pregnancy outcomes in human 8-cell embryos compared to controls.

Keywords: Age; Frozen-thawed embryos; Laser- assisted hatching; Multiple thinning; Pregnancy outcome

\section{Introduction}

Hatching is the event in which the embryo breaks out of the zona pellucida (ZP) during the blastocyst stage, and is very important for successful implantation. Failure of the hatching process has been considered to be a cause of implantation failure in assisted reproductive technology. To overcome failure of the hatching process, a tech-

Received: Sep 16, 2017· Revised: Dec 18, 2017 · Accepted: Jan 23, 2018 Corresponding author: Kyu-Sup Lee

Department of Obstetrics and Gynecology, Pusan National University School of Medicine, 179 Gudeok-ro, Seo-gu, Busan 49241, Korea

Tel: +82-51-240-7287 Fax: +82-51-248-2384 E-mail: kuslee@pusan.ac.kr

*This work was supported by a 2-Year Research Grant from Pusan National University.

This is an Open Access article distributed under the terms of the Creative Commons Attribution Non-Commercial License (http://creativecommons.org/licenses/by-nc/4.0/) which permits unrestricted non-commercial use, distribution, and reproduction in any medium, provided the original work is properly cited. nique in which the ZP is artificially ruptured, known as assisted hatching (AH), has been introduced. However, controversies remain regarding the application of $\mathrm{AH}$.

A review conducted by the American Society for Reproductive Medicine reported that there was no reason to recommend the routine performance of $\mathrm{AH}$ in women undergoing assisted reproductive technology [1]. However, a recent meta-analysis suggested that AH was positively correlated with increased pregnancy rates in women with previous repeated failure or frozen-thawed (FT) embryos [2].

As well as the overall efficacy of $\mathrm{AH}$, several technical aspects of $\mathrm{AH}$ are debatable. A variety of $\mathrm{AH}$ techniques have been introduced since the first report by Cohen et al. [3] in 1988. Initially, AH was performed by thinning or opening the ZP by mechanical [4] or chemical [5] methods. Recently, AH using lasers has become the most popular method because laser AH (LAH) is simple, easy, and rapid [6]. There are also debates on the timing of LAH in terms of the developmental 
stage of the embryo.

The natural hatching process may have polarity, and hatching of the blastocyst may be promoted or inhibited depending on the site of $\mathrm{AH}$. For this reason, side effects due to the incorrect selection of the site for $\mathrm{AH}$ could be a possible problem arising when $\mathrm{AH}$ is performed on a single point of the ZP, since it is difficult to ascertain the correct hatching site at the cleavage stage. Therefore, the determination of the correct AH site, especially in cleavage-stage embryos, may be a very important factor for improving the effectiveness of $\mathrm{AH}$.

Given the above considerations, this study conducted a preliminary investigation of the effect of three-area laser-assisted zona thinning (LAZT) during the cleavage stage on pregnancy outcomes in human in vitro fertilization-embryo transfer (IVF-ET) patients with advanced female age or FT embryos.

\section{Methods}

This study was approved by the Institutional Review Board of Pusan National University Hospital (IRB No. E2016024), Korea, and included 72 consecutive fresh cycles and $28 \mathrm{FT}$ embryo transfer cycles that underwent IVF-ET from March 2015 to December 2015. Informed consent was obtained from all participating patients. In 55 fresh cycles and $17 \mathrm{FT}$ cycles, the patients declined $\mathrm{AH}$ (control group), and day 2 or day 3 embryos underwent LAH in 17 fresh and 11 FT cycles (experimental group).

\section{Controlled ovarian stimulation, oocyte retrieval, embryo culture, and transfer}

IVF was performed by protocols using a gonadotropin-releasing hormone $(\mathrm{GnRH})$ agonist or antagonist with gonadotropins in controlled ovarian stimulation. The choice was based on patient characteristics or response during previous cycles. In the GnRH agonist long protocol, patients were downregulated using a $\mathrm{GnRH}$ agonist (Superfact; Sanofi-Aventis, Frankfurt, Germany) and stimulation with recombinant follicle-stimulating hormone (Gonal-F, Merck Serono, Darmstadt, Germany) was started on the third day of menstruation. When two or more follicles reached $18 \mathrm{~mm}$ in diameter, a dose of $250 \mu \mathrm{g}$ of recombinant human chorionic gonadotropin (Ovidrel, Merck Serono) was administered in both protocols. In the GnRH antagonist protocol, ovarian stimulation was started on the third day of menstruation. When the leading follicles reached a mean diameter of 13-14 mm, a GnRH antagonist (cetrorelix, $0.25 \mathrm{mg} /$ day; Cetrotide, Merck Serono) was administered. If follicular development was slow or insufficient, $75-150 \mathrm{IU} /$ day of recombinant luteinizing hormone (Luveris, Merck Serono) or human menopausal gonadotropin (Menopur; Ferring, Lausanne, Switzerland) was added. When the leading follicles reached a mean diameter of 17-18 mm, a GnRH agonist (triptorelin, $0.2 \mathrm{mg}$; Decapeptyl, Ferring) was administrated to trigger ovulation.

Transvaginal ultrasound-guided oocyte retrieval was carried out 35 hours after human chorionic gonadotropin injection. Oocytes were fertilized in fertilization medium (Sage, Pasadena, CA, USA) and embryos were cultured in cleavage medium (Sage) until day 3 , and then further cultured in blastocyst medium (Sage) until day 5 or 6 . Embryo transfer (ET) was performed on day 3 or 5 . Clinical pregnancy was confirmed by the presence of a gestational sac and a fetal heartbeat on transvaginal ultrasonography 7 weeks after ET.

\section{Embryo freezing and thawing}

Human embryos were frozen at the 2-cell, 4-cell, or 8-cell stage (day 2 or 3) by a slow freezing method. The solutions for cryopreservation and dilution consisted of five graded solutions: solution I, 20\% serum substitute supplement (SSS; Irvine Scientific, Santa Ana, CA, USA) in Dulbecco phosphate-buffered saline (DPBS; Gibco, Waltham, MA USA); solution II, $0.5 \mathrm{M}$ propanediol (PROH) in solution I; solution III, 1.0 M PROH in solution I; solution IV, 1.5 M PROH in solution l; and solution V, 1.5 M PROH+0.2 M sucrose in solution I. The embryos were immersed in each solution for 5 minutes at room temperature and loaded into a 0.25 - $\mathrm{mL}$ plastic straw. They were transferred into an automated Kryo 10 series III biological vertical freezer (Planer, Sunburyon-Thames, UK). The initial chamber temperature was $20^{\circ} \mathrm{C}$, which was then slowly reduced to $-7^{\circ} \mathrm{C}$ at a rate of $2^{\circ} \mathrm{C} / \mathrm{min}$. Ice nucleation was induced manually at $-7^{\circ} \mathrm{C}$. After a holding time of 10 minutes at $-7^{\circ} \mathrm{C}$, the straws were cooled slowly to $-43^{\circ} \mathrm{C}$ at a rate of $0.3^{\circ} \mathrm{C} / \mathrm{min}$ and then rapidly transferred to liquid nitrogen tanks and stored until thawing.

The solutions for thawing consisted of four graded solutions: solution I, 1.0 M PROH+0.2 M sucrose in solution IV; solution II, $0.5 \mathrm{M}$ $\mathrm{PROH}+0.2 \mathrm{M}$ sucrose in solution IV; solution III, $0.2 \mathrm{M}$ sucrose in solution IV; solution IV, $20 \%$ SSS in DPBS. The straws were removed from the liquid nitrogen tanks and air-warmed for 30 seconds at room temperature. The straws were then immersed in a $37^{\circ} \mathrm{C}$ water bath for 40 seconds until all traces of ice disappeared, and the embryos were expelled in solution I. Next, the embryos were placed in each solution for 5 minutes. Finally, the embryos were transferred in culture medium and incubated.

The frozen day 2 or 3 embryos were thawed by a rapid protocol and further cultured before ET. LAZT was performed at the 8-cell stage for both day 3 and day $5 \mathrm{ET}$. In day $3 \mathrm{ET}$, LAZT was performed just before $\mathrm{ET}$, and in day $5 \mathrm{ET}$, LAZT-treated 8-cell embryos were further cultured for 2-3 days. In the 72 cycles of the control group, 46 (63.8\%) were cleavage-stage transfers and 26 (26.2\%) were blastocyst transfers. In the 28 cycles of the LAZT group, 15 (68.1\%) were cleavagestage transfers, and $7(31.8 \%)$ were blastocyst transfers. 


\section{Laser-assisted zona thinning}

LAZT was performed in good-quality 8-cell embryos of grade A and B using OCTAX NaviLase (MTG Medical Technology, Bruckberg, Germany) in three areas of the ZP at a $120^{\circ}$ interval. The original culture dish containing the 8-cell embryos was placed on an inverted microscope equipped with a laser system. The point of treatment on the ZP was carefully focused on and treated with the laser. At that time, the embryos were not stabilized with the holding pipette. One area

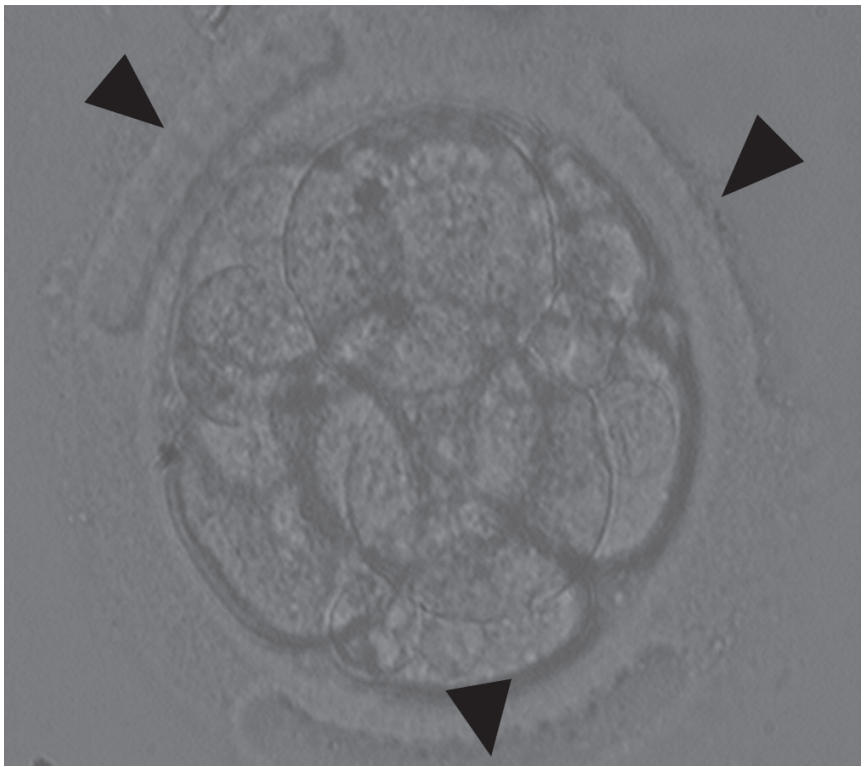

Figure 1. Three-area laser-assisted zona thinning in 8-cell human embryos undergoing in vitro fertilization-embryo transfer. The arrowheads indicate the three areas of zona thinning $(\times 200)$. on the ZP was randomly chosen for LAZT. The ZP was thinned to more than $70 \%$ of its initial thickness using a $1.48 \mu \mathrm{m}$ wavelength diode laser by making 7-8 consequent holes of 15-20 $\mu \mathrm{m}$ on that area of the ZP. All zona thinning was performed in the empty region without contact with the blastomere to minimize the damage. The power of the laser was $100 \%$ and the pulse duration was $500 \mu$ s (Figure 1).

\section{Statistical analysis}

The statistical analyses were performed using IBM SPSS ver. 19.0 (IBM Corp., Armonk, NY, USA) and all data were presented as mean \pm standard deviation. The blastocyst formation rate, hatching rate, and pregnancy outcomes were comparatively analyzed using the chi-square test and $t$-test. The $p$-values $<0.05$ were considered to indicate statistical significance.

\section{Results}

Table 1 shows the clinical characteristics of the patients and the pregnancy outcomes. The pregnancy outcomes were not significantly different between the two groups. No multiple pregnancies were observed in either the control group or the LAZT group.

A total of 79 embryos with 8-cell stage (57 in the control group and 22 in the LAZT group) were further cultured for 2 days, and the formation of expanding blastocysts as well as the early hatching of blastocysts was observed. The blastocyst formation rate was higher in the LAZT group than in the control group, but the difference was statistically insignificant (Table 2).

Of the 72 total cycles, 41 were in women more than 38 years of age.

Table 1. Clinical characteristics and pregnancy outcomes

\begin{tabular}{|c|c|c|c|c|c|}
\hline \multirow{2}{*}{ Variable } & \multicolumn{2}{|c|}{ Control $(n=72)$} & \multicolumn{2}{|c|}{$\operatorname{LAZT}(n=28)$} & \multirow{2}{*}{$p$-value } \\
\hline & Fresh & Frozen-thawed & Fresh & Frozen-thawed & \\
\hline No. of cycles & 55 & 17 & 17 & 11 & - \\
\hline Indication & & & & & - \\
\hline Tubal factor & 27 & 10 & 10 & 6 & \\
\hline Male factor & 8 & 2 & 1 & 1 & \\
\hline Unexplained & 20 & 5 & 6 & 4 & \\
\hline Uterine factor & 0 & 0 & 0 & 0 & \\
\hline Female age (yr) & $34.9 \pm 4.4(28-43)$ & $34.6 \pm 5.0(25-41)$ & $38.8 \pm 5.3(28-48)^{a)}$ & $34.1 \pm 5.0(27-42)$ & $<0.05$ \\
\hline No. of oocytes retrieved & $8.4 \pm 5.9(1-22)$ & $11.3 \pm 5.6(2-24)$ & $5.7 \pm 5.1(1-15)$ & $15.7 \pm 3.7(4-21)$ & NS \\
\hline Fertilization rate (\%) & $82.6 \pm 17.1$ & $81.9 \pm 15.9$ & $83.5 \pm 17.3$ & $84.3 \pm 14.1$ & NS \\
\hline No. of embryos transferred & $2.4 \pm 1.9$ & $2.5 \pm 1.0$ & $2.2 \pm 1.0$ & $2.5 \pm 0.9$ & NS \\
\hline Positive $\beta$-hCG & $17(30.9)$ & $5(29.5)$ & $6(35.2)$ & $5(45.5)$ & NS \\
\hline Clinical pregnancy & $15(27.3)$ & $4(23.5)$ & $3(17.6)$ & $5(45.5)^{b)}$ & $<0.05$ \\
\hline Ongoing pregnancy & $12(21.8)$ & $3(17.6)$ & $2(11.8)$ & $4(36.4)^{b)}$ & $<0.05$ \\
\hline
\end{tabular}

Values are presented as mean $\pm S D$ (range), mean $\pm S D$, or number (\%).

LAZT, laser-assisted zona thinning; NS, not significant; hCG, human chorionic gonadotropin; SD, standard deviation.

${ }^{\text {a) }} p<0.05$ (vs. fresh cycles in the control group); ${ }^{\text {b) }} p<0.05$ (vs. others). 
Table 2. Outcome of blastocyst formation and hatching after LAZT in 8-cell embryos

\begin{tabular}{|c|c|c|c|c|c|}
\hline \multirow{2}{*}{ Variable } & \multicolumn{2}{|c|}{ Control $(n=19)$} & \multicolumn{2}{|c|}{ LAZT $(n=7)$} & \multirow{2}{*}{$p$-value } \\
\hline & Fresh & Frozen-thawed & Fresh & Frozen-thawed & \\
\hline No. of cycles & 13 & 6 & 3 & 4 & - \\
\hline No. of 8-cell embryos & 42 & 15 & 12 & 10 & - \\
\hline Expanding blastocyst & $17(40.5)$ & $6(40.0)$ & $6(50.0)$ & $6(60.0)$ & NS \\
\hline Early hatching blastocyst & $3(7.1)$ & $1(6.7)$ & $2(25.0)$ & $3(33.3)$ & $<0.05^{\mathrm{a})}$ \\
\hline No. of embryos transferred & $1.3 \pm 0.7$ & $1.6 \pm 0.5$ & $1.2 \pm 0.9$ & $1.2 \pm 0.8$ & NS \\
\hline Clinical pregnancy & $4(30.6)$ & $2(33.3)$ & $1(33.0)$ & $2(50.0)$ & NS \\
\hline
\end{tabular}

Values are presented as number (\%) or mean \pm standard deviation.

LAZT, laser-assisted zona thinning; NS, not significant.

${ }^{a)} p$-values were calculated using the chi-square test (vs. control).

Table 3. Effects of LAZT on pregnancy outcomes in patients with advanced female age ( $\geq 38$ years old)

\begin{tabular}{|c|c|c|c|c|c|}
\hline \multirow{2}{*}{ Variable } & \multicolumn{2}{|c|}{ Control $(n=28)$} & \multicolumn{2}{|c|}{$\operatorname{LAZT}(n=13)$} & \multirow{2}{*}{$p$-value ${ }^{\mathrm{a})}$} \\
\hline & Fresh & Frozen-thawed & Fresh & Frozen-thawed & \\
\hline No. of cycles & 18 & 10 & 9 & 4 & - \\
\hline Female age (yr) & $38.9 \pm 1.4$ & $38.4 \pm 2.4$ & $39.7 \pm 3.8$ & $40.4 \pm 2.4$ & NS \\
\hline No. of oocytes retrieved & $6.0 \pm 3.5$ & $6.6 \pm 4.1$ & $4.1 \pm 2.2^{\mathrm{a})}$ & $4.9 \pm 2.9^{\mathrm{a})}$ & $<0.05^{\mathrm{a})}$ \\
\hline No. of embryos transferred & $2.3 \pm 0.7$ & $2.4 \pm 1.0$ & $2.1 \pm 1.2$ & $2.3 \pm 1.3$ & NS \\
\hline Clinical pregnancy & $4(22.2)$ & $2(20.0)$ & $1(11.1)$ & $1(25.0)$ & NS \\
\hline
\end{tabular}

Values are presented as mean \pm standard deviation or number (\%).

LAZT, laser-assisted zona thinning; NS, not significant.

a) $p$-values were calculated using the $t$-test (vs. control).

Table 4. Effects of LAZT on pregnancy outcomes in frozen-thawed cycles

\begin{tabular}{lcc}
\hline Variable & Control & LAZT \\
\hline No. of cycles & 17 & 11 \\
Female age $(y r)$ & $34.6 \pm 5.0$ & $34.1 \pm 5.0$ \\
No. of oocytes retrieved & $11.3 \pm 5.6$ & $15.7 \pm 3.7$ \\
No. of embryos transferred & $2.5 \pm 1.0$ & $2.5 \pm 0.9$ \\
Clinical pregnancy & $4(23.5)$ & $5(45.5)^{\mathrm{a})}$ \\
\hline
\end{tabular}

Values are presented as mean \pm standard deviation or number (\%).

LAZT, laser-assisted zona thinning.

a) $p<0.05$ was calculated using the chi-square test (vs. control).

LAZT was performed in 13 of these cycles. Although the number of oocytes retrieved was significantly lower in the LAZT group than in the control group $(p<0.05)$, both groups showed similar clinical pregnancy rates (Table 3 ).

The clinical pregnancy rate increased significantly to $45.5 \%$ when LAZT was applied in FT cycles compared to the control group (23.5\%) (Table 4), whereas a significant difference was not observed between the control group (27.3\%) and the LAZT group (17.6\%) in the fresh cycles (Table 5). The number of oocytes retrieved and embryos transferred had negligible effects in both groups; however, a significant difference in age was found between the control and LAZT groups in the fresh cycles (Table 5).
Table 5. Effects of LAZT on pregnancy outcomes in fresh cycles

\begin{tabular}{lcc}
\hline Variable & Control & LAZT \\
\hline No. of cycles & 55 & 17 \\
Female age $(y r)$ & $34.9 \pm 4.4$ & $38.8 \pm 5.3^{\text {a) }}$ \\
No. of oocytes retrieved & $8.4 \pm 5.9$ & $5.7 \pm 5.1$ \\
No. of embryos transferred & $2.4 \pm 1.9$ & $2.2 \pm 1.0$ \\
Clinical pregnancy & $15(27.3)$ & $3(17.6)$ \\
\hline
\end{tabular}

Values are presented as mean \pm standard deviation or number (\%). LAZT, laser-assisted zona thinning.

a) $p<0.05$ was calculated using the $t$-test (vs. control).

\section{Discussion}

In this study, LAZT led to significant improvements in pregnancy outcomes in FT embryos, but not in patients with advanced age. This is the first study to demonstrate the efficacy of multi-zone LAZT on embryo development and pregnancy outcomes. This study suggests that an appropriate method is needed to ensure the beneficial effects of $\mathrm{AH}$.

Zona hardening is a process that prevents the natural hatching of blastocysts, and has been known to occur during in vitro culture, after cryopreservation, and in patients with advanced maternal age $[4,7,8]$. For this reason, FT embryos have been considered prime candidates 
for $\mathrm{AH}$ [2], but the benefits of AH in FT embryos have been controversial. Two recent studies showed LAH to have positive effects on clinical pregnancy outcomes $[9,10]$. The present study also showed that LAH could significantly increase the pregnancy rate in FT ET cycles.

However, in this study, the pregnancy rate was unexpectedly higher in the control group than in the LAZT group in the fresh cycles, although this trend was not statistically significant. This primarily means that LAZT had no effect on embryos in fresh cycles. The high pregnancy rate in the control group was probably due to the small sample size, considering that there was no significant difference in the pregnancy rate between the two groups.

Maternal age is another determinant of the use of $\mathrm{AH}$ [11]. Some studies have shown positive effects of $\mathrm{AH}$ when performed in patients younger than 36 years $[12,13]$. However, in other studies, negative or minimal changes in the clinical pregnancy rate were observed in the $\mathrm{AH}$ group compared to the control group in patients younger than 37 years $[14,15]$. Regarding older women, a recent meta-analysis suggested that $\mathrm{AH}$ has little effect on the clinical pregnancy rate in patients with an advanced maternal age of 38 years or older [2]. However, Kanyo et al. [10] reported that LAH significantly increased the pregnancy rate in patients older than 37 years. In this study, LAH did not have different effects on older and younger patients. Regarding this point, it appears that more detailed or homogeneous indications, including female age and the frozen status of the embryos, are important to ensure that $\mathrm{AH}$ is beneficial.

In the present study, LAZT increased the early hatching rate although it was examined in a small number of cases. This effect of LAZT may contribute to the increased pregnancy rate. Miyata et al. [16] showed that hatching was initiated earlier in the AH group than in blastocysts without $\mathrm{AH}$. It has been hypothesized that electrical stimulation may induce egg activation and stimulate embryonic development. Thus, further study is needed to elucidate the correlation between increased hatching rate and pregnancy outcomes after LAZT treatment.

Regarding the hatching site, the findings of this study support the efficacy of multi-area AH. When ET is performed on day 2 or 3 (in 4-cell or 8-cell embryos), the exact site of hatching is unknown. Therefore, zona thinning in three areas, as performed in the present study, could offer a way of increasing the likelihood of hatching. Indeed, the hatching rate in the three-area LAZT group was higher than in the control group in the present study.

The pregnancy rates of day 3 cleavage-stage ET and day 5 blastocyst ET are still controversial, but there is generally no difference between these two groups. Furthermore, the pregnancy rates of day 3 ET and day 5 ET were similar between the control and LAZT groups; therefore, this study did not separately study day 3 ET and day 5 ET.
Thermal damage is an important concern in LAH that has been evaluated in several studies [17-19]. Kanyo and Konc [17] showed that there was no evidence of increased chromosomal abnormalities or congenital malformations in 134 children born after LAH. Zhou et al. [18] reported that no adverse effects were identified in perinatal outcomes after LAH treatment in a retrospective cohort study of a total of 699 women who delivered 392 infants.

This study has several limitations. First, it was conducted retrospectively. Second, the sample size was relatively small. Therefore, the results of this study should be interpreted with caution. However, this is the first study to demonstrate the efficacy of multi-area LAZT on embryo development and pregnancy outcomes. In conclusion, this study showed that LAZT only improved pregnancy outcomes in FT embryos, but not in women with advanced age. These findings should be further evaluated in a prospective randomized study.

\section{Conflict of interest}

No potential conflict of interest relevant to this article was reported.

\section{References}

1. Practice Committee of Society for Assisted Reproductive Technology; Practice Committee of American Society for Reproductive Medicine. The role of assisted hatching in in vitro fertilization: a review of the literature. A Committee opinion. Fertil Steril 2008;90(5 Suppl):S196-8.

2. Martins WP, Rocha IA, Ferriani RA, Nastri CO. Assisted hatching of human embryos: a systematic review and meta-analysis of randomized controlled trials. Hum Reprod Update 2011;17:438-53.

3. Cohen J, Malter H, Fehilly C, Wright G, Elsner C, Kort H, et al. Implantation of embryos after partial opening of oocyte zona pellucida to facilitate sperm penetration. Lancet 1988;2:162.

4. Cohen J, Elsner C, Kort H, Malter H, Massey J, Mayer MP, et al. Impairment of the hatching process following IVF in the human and improvement of implantation by assisting hatching using micromanipulation. Hum Reprod 1990;5:7-13.

5. Cohen J, Alikani M, Trowbridge J, Rosenwaks Z. Implantation enhancement by selective assisted hatching using zona drilling of human embryos with poor prognosis. Hum Reprod 1992;7:68591.

6. Valojerdi MR, Eftekhari-Yazdi P, Karimian L, Ashtiani SK. Effect of laser zona pellucida opening on clinical outcome of assisted reproduction technology in patients with advanced female age, recurrent implantation failure, or frozen-thawed embryos. Fertil Steril 2008;90:84-91.

7. Carroll J, Depypere H, Matthews CD. Freeze-thaw-induced 
changes of the zona pellucida explains decreased rates of fertilization in frozen-thawed mouse oocytes. J Reprod Fertil 1990; 90:547-53.

8. DeMeestere I, Barlow P, Leroy F. Hardening of zona pellucida of mouse oocytes and embryos in vivo and in vitro. Int J Fertil Womens Med 1997;42:219-22.

9. Wan CY, Song C, Diao LH, Li GG, Bao ZJ, Hu XD, et al. Laser-assisted hatching improves clinical outcomes of vitrified-warmed blastocysts developed from low-grade cleavage-stage embryos: a prospective randomized study. Reprod Biomed Online 2014; 28:582-9.

10. Kanyo K, Zeke J, Kriston R, Szucs Z, Cseh S, Somoskoi B, et al. The impact of laser-assisted hatching on the outcome of frozen human embryo transfer cycles. Zygote 2016;24:742-7.

11. Stein A, Rufas O, Amit S, Avrech O, Pinkas H, Ovadia J, et al. Assisted hatching by partial zona dissection of human pre-embryos in patients with recurrent implantation failure after in vitro fertilization. Fertil Steril 1995;63:838-41.

12. Jelinkova L, Pavelkova J, Strehler E, Paulus W, Zivny J, Sterzik K. Improved implantation rate after chemical removal of the zona pellucida. Fertil Steril 2003;79:1299-303.

13. Petersen CG, Mauri AL, Baruffi RL, Oliveira JB, Massaro FC, Elder K, et al. Implantation failures: success of assisted hatching with quarter-laser zona thinning. Reprod Biomed Online 2005;10:224-
9.

14. Primi MP, Senn A, Montag M, Van der Ven H, Mandelbaum J, Veiga $A$, et al. A European multicentre prospective randomized study to assess the use of assisted hatching with a diode laser and the benefit of an immunosuppressive/antibiotic treatment in different patient populations. Hum Reprod 2004;19:2325-33.

15. Rufas-Sapir O, Stein A, Orvieto R, Avrech OM, Kotler N, Pinkas H, et al. Is assisted hatching beneficial in patients with recurrent implantation failures? Clin Exp Obstet Gynecol 2004;31:110-2.

16. Miyata H, Matsubayashi H, Fukutomi N, Matsuba J, Koizumi A, Tomiyama T. Relevance of the site of assisted hatching in thawed human blastocysts: a preliminary report. Fertil Steril 2010;94: 2444-7.

17. Kanyo K, Konc J. A follow-up study of children born after diode laser assisted hatching. Eur J Obstet Gynecol Reprod Biol 2003; 110:176-80.

18. Zhou H, Zao W, Zhang W, Shi J, Shi W. No adverse effects were identified on the perinatal outcomes after laser-assisted hatching treatment. Reprod Biomed Online 2014;29:692-8.

19. Jwa J, Jwa SC, Kuwahara A, Yoshida A, Saito H. Risk of major congenital anomalies after assisted hatching: analysis of three-year data from the national assisted reproduction registry in Japan. Fertil Steril 2015;104:71-8. 